\title{
Elevated Risk for Antimicrobial Drug-Resistant Shigella Infection among Men Who Have Sex with Men, United States, 2011-2015
}

\author{
Anna Bowen, Julian Grass, Amelia Bicknese, Davina Campbell, Jacqueline Hurd, Robert D. Kirkcaldy
}

\begin{abstract}
Shigella spp. cause $\approx 500,000$ illnesses in the United States annually, and resistance to ciprofloxacin, ceftriaxone, and azithromycin is emerging. We investigated associations between transmission route and antimicrobial resistance among US shigellosis clusters reported during 2011-2015. Of 32 clusters, 9 were caused by shigellae resistant to ciprofloxacin (3 clusters), ceftriaxone (2 clusters), or azithromycin ( 7 clusters); 3 clusters were resistant to $>1$ of these drugs. We observed resistance to any of these drugs in all 7 clusters among men who have sex with men (MSM) but in only 2 of the other 25 clusters $(p<0.001)$. Azithromycin resistance was more common among MSM-associated clusters than other clusters ( $86 \%$ vs. $4 \%$ of clusters; $p<0.001)$. For adults with suspected shigellosis, clinicians should culture feces; obtain sex histories; discuss shigellosis prevention; and choose treatment, when needed, according to antimicrobial drug susceptibility. Public health interviews for enteric illnesses should encompass sex practices; health messaging for MSM must include shigellosis prevention.
\end{abstract}

Shigellosis, the third most common human bacterial enSteric infection in the United States, causes $\approx 500,000$ illnesses each year (1). Because as few as 10 bacteria can cause infection, shigellosis outbreaks typically are large; control can be difficult unless interventions are implemented early in an outbreak (2). Although bloodstream infection is uncommon among immunocompetent hosts, shigellosis patients frequently are treated with antimicrobial medications to reduce illness duration and possibly transmission (3). High rates of resistance to ampicillin and trimethoprim/ sulfamethoxazole have made ciprofloxacin, ceftriaxone, and azithromycin the preferred antimicrobial agents for adults and children with shigellosis; ceftriaxone is also the preferred treatment for invasive shigellosis (4-6). However, shigellae resistant to these drugs have emerged in the United States and abroad (7-17). Although shigellosis rates are highest for young children, most reports document

Author affiliation: Centers for Disease Control and Prevention, Atlanta, Georgia, USA

DOI: http://dx.doi.org/10.3201/eid2209.160624 ciprofloxacin- or azithromycin-resistant shigellosis largely among men who have sex with men (MSM) $(7,8,10,12$ 17). Estimates of risk for antimicrobial drug-resistant shigellae among different populations or by transmission route have not been reported. We investigated the associations between transmission route and antimicrobial resistance among US shigellosis clusters reported during 2011-2015.

\section{Methods}

Because sexual transmission data are not captured in the national enteric disease surveillance systems of the Centers for Disease Control and Prevention (CDC), we queried the enteric disease cluster management database of the CDC Outbreak Response and Prevention Branch (ORPB; Division of Foodborne, Waterborne, and Environmental Diseases, National Center for Emerging and Zoonotic Infectious Disease) for all US shigellosis clusters during January 2011-December $2015(18-21)$. This database is not part of a surveillance system; instead, it is used in real time to track and guide response to clusters of enteric infections, including shigellosis, that $\mathrm{CDC}$ investigates. It contains unpublished summaries of patient demographics, suspected or confirmed transmission route and vehicles, cluster onset date and duration, and pulsed-field gel electrophoresis (PFGE) patterns associated with the cluster. ORPB identifies shigellosis clusters for inclusion in this database in 3 ways: 1) when CDC's PulseNet observes a Shigella PFGE pattern approximately twice as often as baseline and in $>1$ US state or territory during a 60day period, PulseNet assigns a cluster code to that pattern; 2) state health departments can choose to contact CDC about ongoing single-state or multistate clusters; 3) since 2014, CDC also has used data from the National Antimicrobial Resistance Monitoring System for Enteric Bacteria (NARMS), which strives to test every 20th Shigella isolate nationally and 3 representative isolates from every shigellosis outbreak, to identify isolates harboring resistance to clinically important antimicrobials $(22,23)$. CDC then queries PulseNet for other isolates with PFGE patterns indistinguishable from the resistant isolate. For Shigella, all identified clusters are added to the ORPB database. 
Our analysis comprised clusters with both antimicrobial drug susceptibility data in NARMS and documentation of transmission route or affected population. We linked clusters to NARMS by PulseNet cluster code or local outbreak code to obtain information about the antimicrobial resistance phenotype associated with the cluster. We used standard definitions for ciprofloxacin and ceftriaxone resistance and defined azithromycin resistance as an azithromycin MIC greater than that for wild-type Shigella (24). Among isolates with phenotypic azithromycin resistance, we used PCR to screen for the macrolide resistance genes $m p h \mathrm{~A}$ and $e r m \mathrm{~B}$. We classified a cluster as resistant to $\geq 1$ of these antimicrobial drugs if any isolate within the cluster harbored resistance to $\geq 1$ of these drugs.

Few public health jurisdictions routinely collect the MSM status of shigellosis patients, but they might do so through telephone interviews, in-person interviews, medical record reviews, or links to sexual health databases. Public health jurisdictions did not use standardized definitions for MSM-associated transmission; however, we categorized transmission as MSM-associated if any MSM-associated transmission was recorded for a cluster. If subclusters with distinct transmission routes were reported within a single cluster, we analyzed the subclusters as separate clusters. Using Fisher exact test, we compared proportions of clusters with antimicrobial resistance by transmission category.

\section{Results}

Of 49 clusters reported during 2011-2015, we excluded 19 (39\%) because of insufficient data (transmission data but no antimicrobial resistance data, 5 clusters; neither transmission data nor antimicrobial resistance data, 14 clusters). Of the $30(61 \%)$ remaining clusters, 2 encompassing subclusters with MSM-associated and other person-to-person transmission were further divided by transmission category, for a total of 32 clusters eligible for analysis (Table 1, http://wwwnc.cdc.gov/EID/article/22/9/16-0624-T1.htm). Suspected or confirmed transmission was reported to involve child care centers, camps, or schools (10 clusters); MSM (7 clusters); other person-to-person transmission (7 clusters); food (6 clusters); or recreational water (2 clusters). Nine clusters were caused by shigellae resistant to at least 1 of the following: ciprofloxacin (3 clusters), ceftriaxone ( 2 clusters), or azithromycin ( 7 clusters). Three of these clusters were resistant to $>1$ of these drugs. At least 1 isolate from each azithromycin-resistant cluster was confirmed to harbor the macrolide resistance genes $m p h \mathrm{~A}$ or ermB. Of the 9 antimicrobial drug-resistant clusters, 8 were caused by $S$. sonnei and 1 by $S$. flexneri. Isolates from all $7 \mathrm{MSM}$-associated clusters harbored resistance to $\geq 1$ of these drugs (Table 1). The prevalence of resistance among MSM-associated and other clusters significantly differed for ceftriaxone $(p=0.04)$, azithromycin $(p<0.001)$, azithromycin and either ciprofloxacin or ceftriaxone $(\mathrm{p}=$ $0.007)$, and any of these 3 drugs $(p<0.001)$ (Table 2). The proportion of MSM-associated clusters with resistance to any of these 3 drugs (i.e., ciprofloxacin, ceftriaxone, or azithromycin) was 3-77 times greater, and the proportion with resistance to azithromycin was 3-500 times greater, than the proportion of other clusters with such resistance phenotypes. None of 10 clusters associated with child care, camps, or schools was caused by ciprofloxacin-, ceftriaxone-, or azithromycin-resistant strains.

\section{Discussion}

All shigellosis clusters that we identified among MSM in the United States during 2011-2015 were caused by strains resistant to $\geq 1$ of the preferred antimicrobial agents for shigellosis. Although our sample was small, the estimated prevalence of resistance to preferred antimicrobial drugs for MSM-associated shigellosis clusters was 3-77 times the prevalence for clusters with nonsexual transmission routes. Although shigellosis with these antimicrobial drug resistance phenotypes has been documented among MSM internationally, the reasons for this association are unknown $(7,8,12-14,16,17)$. Additional studies are needed to elucidate these findings; identify specific risk factors; understand clinical outcomes for patients infected with these resistant strains and in the setting of HIV infection; and develop effective interventions to prevent infection of MSM with shigellae, particularly drug-resistant shigellae.

Although our results suggest that shigellae with resistance to ciprofloxacin, ceftriaxone, or azithromycin circulate predominantly among MSM in the United States, these

\begin{tabular}{|c|c|c|c|}
\hline Antimicrobial resistance phenotype & $\begin{array}{c}\text { MSM-associated transmission, } \\
\text { no. }(\%, 95 \% \mathrm{Cl}+), \mathrm{n}=7\end{array}$ & $\begin{array}{c}\text { Transmission other than MSM- } \\
\text { associated, no. }(\%, 95 \% \mathrm{Cl} \dagger), \mathrm{n}=25\end{array}$ & $p$ valuef \\
\hline CIP & $2(29,5-67)$ & $1(4,0.2-18)$ & 0.1 \\
\hline $\mathrm{CRO}$ & $2(29,5-67)$ & $0(0,0-11)$ & 0.04 \\
\hline AZM & $6(86,47-99)$ & $1(4,0.2-18)$ & $<0.001$ \\
\hline AZM, CIP, or CRO & $7(100,65-100)$ & $2(8,1.3-24)$ & $<0.001$ \\
\hline AZM and either CIP or CRO & $3(43,12-78)$ & $0(0,0-11)$ & 0.007 \\
\hline
\end{tabular}

AZM and either

$3(43,12-78)$

$<0.001$

*AZM, azithromycin; CIP, ciprofloxacin; CRO, ceftriaxone; MSM, men who have sex with men.

†Mid-p exact $95 \% \mathrm{Cl}$ of the percentage resistant.

‡By 2-tailed Fisher exact test. 
strains are likely to emerge among other populations. Efforts to facilitate improved hygiene practices among persons at high risk for shigellosis or at high risk for transmitting shigellosis to others (e.g., child care attendees, staff, and parents; marginally housed persons; international travelers; and food handlers) are needed now to limit transmission when multidrug-resistant Shigella strains inevitably begin circulating among these populations.

Although the associations we found between antimicrobial drug-resistant shigellosis and transmission route (i.e., MSM-associated transmission vs. other transmission) are striking, this analysis has several limitations. Because the ORPB cluster management database is used to guide response rather than as a formal reporting system, it is likely to contain only a small fraction of shigellosis clusters, and the details about each cluster are not always complete. Furthermore, many public health jurisdictions do not routinely perform PFGE on shigellae, making it difficult to detect clusters and, when a cluster is detected, to locate all cases associated with the cluster. Therefore, clusters included in this analysis might not be representative of all US shigellosis clusters, and the number of cases reported per cluster is likely to be smaller than reality. Additionally, nearly $40 \%$ of clusters were not analyzed because of missing data. However, the selection of clusters in this analysis is unlikely to have biased the association between antimicrobial drug resistance phenotype and transmission route. Next, both antimicrobial drug resistance phenotypes and transmission routes are likely to be heterogeneous within each cluster. We also lacked information about patients' antecedent exposure to antimicrobial drugs, HIV infection, and other factors that might enhance our findings, and we did not have access to clinical treatment or outcome data. Finally, because of the small sample size, we cannot reliably compare the prevalence of resistance among MSM-associated clusters and other clusters. Nonetheless, the markedly higher prevalence of such resistance observed in our study is concerning and warrants future study.

The increasing use of PCR-based, culture-independent diagnostic tests for Shigella will make it difficult to identify cases and clusters of multidrug-resistant shigellosis and to provide laboratory-informed antimicrobial treatment (25). When shigellosis is suspected or when a culture-independent diagnostic test suggests Shigella infection, clinicians should culture fecal specimens and test Shigella isolates for antimicrobial drug susceptibility, including susceptibility to azithromycin (24). Patients who do not improve with treatment should be re-cultured. Further characterizing isolates by PFGE or whole-genome sequencing will assist health departments with cluster detection and control.

Alternative treatment options are limited for persons infected with ciprofloxacin-, ceftriaxone-, or azithromycinresistant shigellae, making shigellosis prevention critical.
Clinicians should counsel all patients with suspected shigellosis about meticulous handwashing and hygiene (26). Among adult patients, sexual histories focused on sex of recent partners and specific sex practices can inform empiric treatment and indicate a need for further testing and counseling to prevent sexual transmission of shigellosis (27). If treatment is required, drug choice should be informed by the results of antimicrobial susceptibility testing. During public health interviews for enteric illnesses, routinely collecting sex of sex partners and, among those with multiple recent partners, where patients find partners would improve understanding of risk groups and permit more targeted interventions. Surveillance and interventions for shigellosis among MSM would be strengthened by more intensive collaboration between enteric disease and sexually transmitted disease programs within public health departments. Health messaging for MSM should include information about risk for and prevention of shigellosis and drug-resistant shigellosis $(26,27)$.

\section{Acknowledgments}

We thank Thai-An Nguyen and Sunkyung Kim for reviewing the manuscript.

A.B. had full access to all data in the study and takes responsibility for the integrity of the data and the accuracy of the data analysis, study concept and design, drafting of the manuscript, statistical analysis, and study supervision. All authors contributed to data acquisition, analysis, or interpretation.

Dr. Bowen is a medical epidemiologist in CDC's

Waterborne Disease Prevention Branch, Division of Foodborne, Waterborne, and Environmental Diseases, National Center for Emerging and Zoonotic Infectious Disease. Her research interests include diarrheal disease, child health, handwashing, and antimicrobial resistance.

\section{References}

1. Scallan E, Hoekstra RM, Angulo FJ, Tauxe RV, Widdowson MA, Roy SL, et al. Foodborne illness acquired in the United States - major pathogens. Emerg Infect Dis. 2011;17:7-15. http://dx.doi.org/10.3201/eid1701.P11101

2. DuPont HL, Levine MM, Hornick RB, Formal SB. Inoculum size in shigellosis and implications for expected mode of transmission. J Infect Dis. 1989;159:1126-8. http://dx.doi.org/10.1093/ infdis/159.6.1126

3. Christopher PR, David KV, John SM, Sankarapandian V. Antibiotic therapy for Shigella dysentery. Cochrane Database Syst Rev. 2010; (8):CD006784

4. Kimberlin DWBM, Jackson MA, Long SS, editors. Shigella infections. In: Red book: 2015 report of the Committee on Infectious Diseases. 30th ed. Elk Grove (IL): American Academy of Pediatrics; 2015. p. 706-9.

5. Gilbert DN, editor. Sanford guide to antimicrobial therapy. 45th ed. Sperryville (VA): Antimicrobial Therapy, Inc.; 2015.

6. Serafino Wani RL, Filson SA, Chattaway MA, Godbole G. Invasive shigellosis in MSM. Int J STD AIDS. 2015;Oct 1:pii: 0956462415610275 . 
7. Baker KS, Dallman TJ, Ashton PM, Day M, Hughes G, Crook PD, et al. Intercontinental dissemination of azithromycin-resistant shigellosis through sexual transmission: a cross-sectional study. Lancet Infect Dis. 2015;15:913-21. http://dx.doi.org/10.1016/ S1473-3099(15)00002-X

8. Bowen A, Eikmeier D, Talley P, Siston A, Smith S, Hurd J, et al.; Centers for Disease Control and Prevention (CDC). Notes from the field: outbreaks of Shigella sonnei infection with decreased susceptibility to azithromycin among men who have sex with men-Chicago and metropolitan Minneapolis-St. Paul, 2014. MMWR Morb Mortal Wkly Rep. 2015;64:597-8.

9. Bowen A, Hurd J, Hoover C, Khachadourian Y, Traphagen E, Harvey E, et al.; Centers for Disease Control and Prevention (CDC). Importation and domestic transmission of Shigella sonnei resistant to ciprofloxacin-United States, May 2014-February 2015. MMWR Morb Mortal Wkly Rep. 2015;64:318-20.

10. Centers for Disease Control and Prevention. HAN 379: ciprofloxacin- and azithromycin-nonsusceptible shigellosis in the United States [cited 2016 Jan 20]. http://content.govdelivery.com/ accounts/USCDC/bulletins/107d50c

11. Centers for Disease Control and Prevention (CDC). Notes from the field: Outbreak of infections caused by Shigella sonnei with decreased susceptibility to azithromycin-Los Angeles, California, 2012. MMWR Morb Mortal Wkly Rep. 2013;62:171.

12. Chiou CS, Izumiya H, Kawamura M, Liao YS, Su YS, Wu HH, et al. The worldwide spread of ciprofloxacin-resistant Shigella sonnei among HIV-infected men who have sex with men, Taiwan. Clin Microbiol Infect. 2016;22:383.e11-6. http://dx.doi.org/10.1016/j.cmi.2015.12.021

13. Gaudreau C, Barkati S, Leduc JM, Pilon PA, Favreau J, Bekal S. Shigella spp. with reduced azithromycin susceptibility, Quebec, Canada, 2012-2013. Emerg Infect Dis. 2014;20:854-6. http://dx.doi.org/10.3201/eid2005.130966

14. Gaudreau C, Ratnayake R, Pilon PA, Gagnon S, Roger M, Lévesque S. Ciprofloxacin-resistant Shigella sonnei among men who have sex with men, Canada, 2010. Emerg Infect Dis. 2011;17:1747-50. http://dx.doi.org/10.3201/eid1709.102034

15. Heiman KE, Karlsson M, Grass J, Howie B, Kirkcaldy RD, Mahon B, et al.; Centers for Disease Control and Prevention (CDC). Notes from the field: Shigella with decreased susceptibility to azithromycin among men who have sex with men-United States, 2002-2013. MMWR Morb Mortal Wkly Rep. 2014;63:132-3.
16. Hoffmann C, Sahly H, Jessen A, Ingiliz P, Stellbrink HJ, Neifer S, et al. High rates of quinolone-resistant strains of Shigella sonnei in HIV-infected MSM. Infection. 2013;41:999-1003. http://dx.doi.org/10.1007/s15010-013-0501-4

17. Lane CR, Sutton B, Valcanis M, Kirk M, Walker C, Lalor K, et al. Travel destinations and sexual behavior as indicators of antibiotic resistant Shigella strains - Victoria, Australia. Clin Infect Dis. 2016;62:722-9. http://dx.doi.org/10.1093/cid/civ1018

18. Centers for Disease Control and Prevention. National Outbreak Reporting System [cited 2016 Feb 29]. http://www.cdc.gov/nors/

19. Centers for Disease Control and Prevention. National surveillance of bacterial foodborne illness (enteric diseases) [cited 2016 Feb 29]. http://www.cdc.gov/nationalsurveillance/shigella-surveillance.html

20. Centers for Disease Control and Prevention. National Notifiable Diseases Surveillance System (NNDSS) [cited 2016 Feb 29]. http://wwwn.cdc.gov/nndss/

21. Centers for Disease Control and Prevention. Outbreak Response Team [cited 2016 Jan 20]. http://www.cdc.gov/ncezid/dfwed/orpb/ ort.html

22. Centers for Disease Control and Prevention. PulseNet [cited 2016 Feb 29]. http://www.cdc.gov/pulsenet/

23. Centers for Disease Control and Prevention. National Antimicrobial Monitoring System for Enteric Bacteria (NARMS) [cited 2016 Feb 29]. http://www.cdc.gov/narms/index.html

24. Clinical and Laboratory Standards Institute. Performance standards for antimicrobial susceptibility testing. Wayne (PA): The Institute; 2016.

25. Iwamoto M, Huang JY, Cronquist AB, Medus C, Hurd S, Zansky S, et al.; Centers for Disease Control and Prevention (CDC). Bacterial enteric infections detected by culture-independent diagnostic tests - FoodNet, United States, 2012-2014. MMWR Morb Mortal Wkly Rep. 2015;64:252-7.

26. Centers for Disease Control and Prevention. Shigella-shigellosis: prevention and control [cited 2016 Feb 29]. http://www.cdc.gov/ shigella/prevention-control.html

27. Centers for Disease Control and Prevention. Shigella infections among gay and bisexual men [cited 2016 Jan 29]. http://www.cdc.gov/shigella/msm.html

Address for correspondence: Anna Bowen, Centers for Disease Control and Prevention, 1600 Clifton R NE, Mailstop C09, Atlanta, GA 303294027, USA; email: abowen@cdc.gov

\title{
gOVDELIVERY \\ Manage your email alerts so you only receive content of interest to you.
}

\author{
Sign up for an online subscription: \\ wwwnc.cdc.gov/eid/subscribe.htm
}

